\title{
Recherche et localisation de fuites sur retenues naturelles ou artificielles par techniques de traceurs
}

PAR

\author{
J. Molinari \\ Ingénieur an Commissariat is l'Energie Atomique $\left({ }^{*}\right)$
}

\section{Introduction}

La maitrise de l'utilisation des ressources en eau, tant pour l'irrigation que pour la production d'énergie, impose l'exploitation de réservoirs naturels ou l'aménagement de rétenues artificielles pour lesquelles, sauf circonstances exceptionnelles, se posent des problèmes d'étanchéité assortis de pertes financières ou de risques rarement négligeables.

On estimait en effet en 1970 à plus de 10000000 de francs le manque à gagner capitalisé d'une fuite de $1 \mathrm{~m}^{3} / \mathrm{s}$ sous 100 mètres de dénivelée, et, dans une étude de synthèse sur l'inspection et la surveillance des barrages diffusée à la même époque [1], on faisait par allleurs état de statistiques montrant que si, au cours des 8 derniers siècles, périssait dans le monde en moyenne un barrage tous les cinq ans, depuis quarante années, la fréquence de ces catastrophes s'était considérablement accrue puisque l'on observait maintenant une rupture de barrage tous les quinze mois.

Préoccupations de cout ou de sécurité conduisent aujourd'hui donc à des investigations, souvent laborieuses et toujours complexes, impliquant un contrôle méticuleux des fuites et réservant quelquefois à leur recherche et à leur localisation une place prépondérante.

Le texte qui va suivre expose les procédés de recherche et de localisation de fuites faisant appel aux techniques souvent méconnues d'identification par traceurs naturels ou artificiels.

Une telle méconnaissance de ces moyens, qui consistent à observer précisément le phénomène préoccupant, n'est pas

(*) Service d'Applications des Radioéléments et des Rayonnements Centre d'Etudes Nucléaires de Grenoble 85 X - 38041 Grenoble Cédex surprenante si l'on tient compte de la relative modernité de techniques qui ont connu leur essor au cours des dernières décennies et qui font quelquefois appel à des procédés de mesure et des méthodes d'interprétation pratiqués dans des disciplines scientifiques bien éloignées de l'hydraulique ou de l'hy drologie.

\section{Investigations préliminaires}

\subsection{OBSERVATIONS VISUELLES}

Ces observations constituent un élément essentiel des investigations. L'expérience montre en outre que sur la plupart des sites, faute d'examen détaillé, des résurgences ou des zones d'infiltration restent indécelées jusqu'à la date des interventions.

De telles omissions privent l'exploitant et l'investigateur de données précieuses, contraignent à des travaux d'aménagement improvisés et compromettent le bon déroulement des essais.

Dans la reconnaissance du site qui s'effectuera si possible dans plusieurs circonstances hydrométéorologiques distinctes, on portera son attention, en aval du réservoir, aux suintements, aux anomalies de la couverture végétale qu'il faudra quelquefois dégager et on recherchera, dans les thalwegs et le cours des torrents, d'éventuelles venues d'eau (repérables par des turbulences en eaux calmes ou détectables par les mesures évoquées plus loin).

Dans la retenue, on s'efforcera de déceler des courants établis (entonnements ou vortex) et, lors des étiages ou des vidanges, fissures, éboulements ou accumulation de débris végétaux. 


\subsection{CONTROLES SYSTEMATIQUES}

\subsubsection{Contróle des fuites}

Sur les retenues artificielles, les drains, dont on devra s'assurer du bon état, peuvent accuser des débits anormaux, indices de fuites à travers l'ouvrage, alors que les fuites qui apparaissent à la base d'un ouvrage sont souvent l'indice de liaisons défectueuses entre l'ouvrage et sa fondation et que celles qui se manifestent au droit du thalweg ou sur les rives peuvent résulter d'infiltrations à travers le terrain lui-même.

Il importe, dans tous les cas, de procéder à l'inventaire exhaustif de toutes les émergences, de connaître leur débit et d'en apprécier les variations. Ce dernier point est particulièrement important compte tenu de la variation importante du débit de fuite en fonction de la charge, l'allure des courbes pouvant renseigner sur la nature des circulations et la cote des infiltrations.

On notera que les émergences pouvant etre influencées par des apports superficiels d'eaux météoriques ou souterrains d'eaux ne provenant pas de la retenue, on ne peut pas toujours, à ce stade des investigations, évaluer l'importance réelle des débits de fuite. Cette discrimination sera d'autant plus facilitée que l'on aura pris le soin de procéder aux mesures de débit avec une fréquence suffisante tenant compte des conditions climatiques et de les compléter par d'autres observations (cf. 2.3).

\subsubsection{Piézométrie}

En complément des observations précitées, la piézométrie précise la forme de la surface libre d'écoulement ou la répartition de sous pressions dans le corps de l'ouvrage et ses appuis.

\subsubsection{Observations hydrométéorologiques - Quelques rap- pels de limnologie}

Outre l'incidence possible des précipitations sur les débits mesurés aux divers exutoires (cf 2.2.1), des observations météorologiques suivies sont indispensables pour interpréter correctement :

-- les mesures isotopiques (cf. 3.2.3)

- la thermométrie (cf. 2.2.4), étroitement liée à la limnologie dont il faut rappeler ici quelques données essentielles

Les contrastes climatiques et météorologiques observés dans la plupart des régions du globe provoquent, généralement dans les retenues d'importance notable, une stratification des eaux résultant des écarts de densité entre eaux de températures différentes (*).

On distingue généralement dans des retenues deux couches de densité et de températures différentes : l'hypolimnion et l'épilimnion séparés par une couche de transitions, la thermocline.

Dans la plupart des retenues situées dans des régions tempérées à climat contrasté, au cours de la saison chaude, l'épilimnion s'épaissit aux dépends de l'hypolimnion froid, la cote de la thermocline s'abaissant vers le fond de la retenue. Inversement, l'hiver, l'hypolimnion, maintenu à la température de densité maximale de l'eau ( $\sim 4$ degrés), est coiffé par les eaux plus froides mais plus légères de l'épilimnion.

Aux deux saisons de transition, l'automne et le printemps, l'égalisation des températures des deux couches provoque

(*) Dans certaines circonstances exceptionnelies, à ces causes thermiques peuvent se superposer des effets de salinité (halocline) ou de turbidité. le brassage total des eaux de la retenue (circulations automnale et vernale). Ces retenues sont qualifiées de bimictiques. Dans certaines contrées, on n'observe qu'un seul brassage annuel, le réservoir est alors qualifié de monomictique.

\subsubsection{Températures comparées de la retenue et des émergences}

L'évolution comparée de la température des eaux de la retenue et de celles des émergences éclaire sur l'origine et la nature des circulations.

On tiendra compte du fait que les phénomènes à analyser (amplitude des variations de température et retard) peuvent être, dans certains types d'ouvrage comme les barrages à enrochements, sensiblement affectés par des variations rapides de température extérieure qu'il semble prudent de relever.

\subsection{TRAITEMENT DES DONNEES}

On expose en 4.1.4. le cas d'émergences de fuites influencées par des apports météoriques et dont les débits de fuite restaient entachés d'imprécision résultant de l'importance et des fluctuations des apports. Comme l'on dispose généralement d'importantes séries statistiques sur ce type de données hydrométriques et hydrogéologiques, il s'avère extrêmement intéressant d'appliquer à ces données un programme de recherches de corrélations multilinéaires de mise en ceuvre et d'interprétation aisée.

On a en effet, obtenu dans l'exemple précédemment cité (barrage d'Alfeld), des relations de corrélation spatiales, spatiotemporelles et phénoménologiques (hauteurs de précipitations).

L'interprétation de ces résultats a permis :

1) de disposer de séries statistiques plus complètes affranchies du "bruit de fond météorique", en particulier au cours des opérations cruciales de remplissage-vidange du réservoir. lors du tracé des diagrammes "débits de fuite-charge de la retenue".

2) d'analyser les comportements des différents massifs drainants et leurs contributions respectives en vue d'éventuels remaniements des installations de contrôle et de surveillance.

3) d'envisager, en phase finale de l'étude, la mise en place d'un dispositif "différentiel" d'alerte utilisant les informations collectées d'une part par un pluviographe enregistreur, d'autre part par des débitmètres, afin de déceler toute augmentation intempestive des débits réels de fuite.

\section{Informations fournies par les indicateurs présents dans le milieu naturel}

\subsection{METHODOLOGIE}

Les concentrations spatiales relatives en indicateurs naturels ainsi que leurs variations au cours du temps permettent d'établir ou de réfuter l'existence de relations entre la retenue et des exurgences et, éventuellement, de préciser certaines modalités de transit.

Les observations à effectuer dans la retenue tiendront compte de l'existence éventuelle d'une stratification d'eaux de température, de teneurs en sédiments ou en colloïdes, de compositions chimique ou isotopique différentes. Elles seront nécessairement complétées par des observations à effectuer 
sur des eaux de précipitations, des eaux de surface ou des eaux souterraines du site environnant (fig. 1).

En général, les processus de transit par des circulations privilégiées à travers un ouvrage ou ses appuis sont suffisamment rapides vis-à-vis des fluctuations des signaux d'entrée pour que des mesures d'une campagne d'échantillonnage extemporanée, entreprises à une date judicieuse, soient interprétables directement pour, comme le montrent les exemples suivants (cf. 3.3):

a) établir des présomptions sur l'existence ou l'absence de circulations directes réservoir-résurgences ;

b) localiser approximativement les cotes d'infiltration.

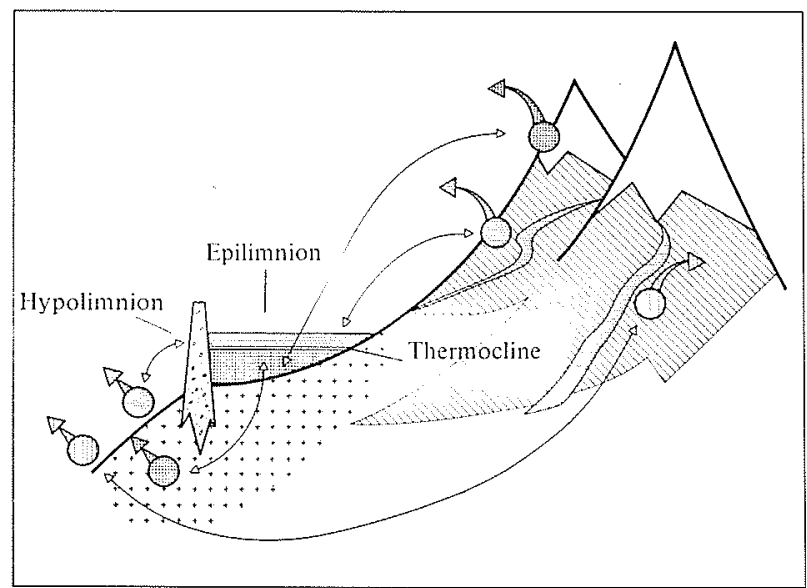

$1 /$ Informations fournies par les indicateurs présents dans le milieu naturel. Exemple de corrélations retenue-résurgences.

Des mesures renouvelées sur des périodes d'observations de longue durée (une année hydrométéorologique au moins) fournissent des informations plus rigoureuses et considérablement plus riches au point que l'on puisse envisager, dans certains cas où les signaux d'entrée et de sortie sont suffisamment contrastés, de calculer la fonction d'intercorrélation qui les relie, fonction représentative des modalités de transfert.

Toutes ces informations sont obtenues sans autre intervention qu'un échantillonnage simple et peu coûteux.

\subsection{CONTROLES ANALYTIQUES DES INDICATEURS NATURELS}

\subsubsection{Matières en suspension}

Le contrôle des matières en suspension, qui peuvent résulter de l'érosion de l'ouvrage ou provenir de certaines strates de la retenue, éclaire sur les effets ou la nature des circulations.

\subsubsection{Géochimie des eaux}

Les analyses chimiques classiques d'éléments majeurs (diagrammes de Schoeller Berkaloff) gagnent à être complétées par des analyses d'éléments trace. L'interprétation de leurs concentrations relatives et absolues fournit généralement des indices intéressants sur l'origine des circulations et surtout sur l'importance de l'altération éventuelle de l'assise ou du corps des ouvrages, si l'on accorde une attention particulière aux éléments susceptibles de caractériser la présence de matériaux constructifs allogènes $(*)$

$\left(^{*}\right)$ Les mesures de rapport isotopiques ${ }^{32} \mathrm{~S} /{ }^{34} \mathrm{~S}$ dans les sulfates ou sulfures peuvent notamment révéler la présence de produits d'altération de ciments ou liants.

\subsubsection{Géochimie isotopique}

Les indicateurs naturels dont on tire les informations les plus intéressantes sont les variétés isotopiques de l'eau : HDO, HTO et $\mathrm{H}_{2}{ }^{18} \mathrm{O}$, idéalement représentatives du mouvement de l'eau [2] qui présentent des variations de concentration spatiales (avec l'altitude), saisonnières (avec les températures) et temporelles (tritium par sa décroissance radioactive). De surcroit, l'étude des corrélations $D /{ }^{18} \mathrm{O}$ permet de distinguer, dans des eaux de même provenance, celles qui ont été soumises à évaporation (eaux de surface) $[2,3]$.

\subsection{EXEMPLES}

\subsubsection{Détermination d'une cote d'infiltration}

L'étroite parenté entre les teneurs en deutérium des eaux de surface de la retenue d'Alfeld [4] et les eaux d'une des cinq émergences situées à son pied a conduit à émettre l'hypothèse que, pour cette émergence particulière, les eaux d'apport pourraient provenir à la fois des eaux de précipitations (comme l'avaient établi de nombreuses autres observations) et des eaux superficielles de la retenue, par l'intermédiaire de l'un des déversoirs de crue.

Cette hypothèse a été confirmée à l'occasion d'une intervention sur le déversoir incriminé, sa mise hors service s'étant, en effet, accompagnée d'une réduction sensible des débits de l'émergence intéressée.

\subsubsection{Etude des modalités de transit à travers les appuis d'un ouvrage [5]}

La mise en eau du barrage de Sidi Messaoud sur l'oued Nebaana s'était accompagnée d'une augmentation de débit d'une source (aïn El Kebrit) située au pied du massif d'appui Rive Gauche et d'apparition d'émergences dans cette même zone ainsi que le long d'un thalweg (oued Aïch) situé en Rive Droite de la retenue (Fig. 2).

En raison de l'importance du débit de ces exutoires sauvages par rapport au module annuel des apports (environ $30 \%$ ), des contrôles et des investigations ont été entrepris, dès le début de la mise en eau de la retenue, pour répondre au souci de préciser la nature des circulations souterraines nouvellement apparues, d'établir la matérialité des fuites et, éventuellement, d'y remédier.

On notera que la topographie des lieux, relativement favorable en Rive Droite où la collecte gravitaire des exutoires de l'oued Aïch se révélait aisée, s'avérait préoccupante en Rive Gauche où une opération semblable, imposée par l'importance des débits, nécessitait des travaux de génie civil considérables.

Dans ce dernier cas, l'interception des circulations souterraines par un masque paraissait devoir être plus économique, d'autant que l'étude géologique avait signalé l'existence d'une faille Sud-Nord à laquelle pouvaient être imputées les circulations et susceptible d'être aisément étanchée.

En fait, un échantillonnage extemporané dans la retenue et les émergences a suffi pour établir, qu'à l'exception d'une seule émergence située en Rive Gauche, tous les prélèvements avaient des teneurs en tritium et/ou en deutérium différentes de celles de la retenue, et que le transit entre la retenue et les émergences ne pouvait s'effectuer par une circulation privilégiée imputable à l'accident tectonique invoqué plus haut (Fig. 3). 


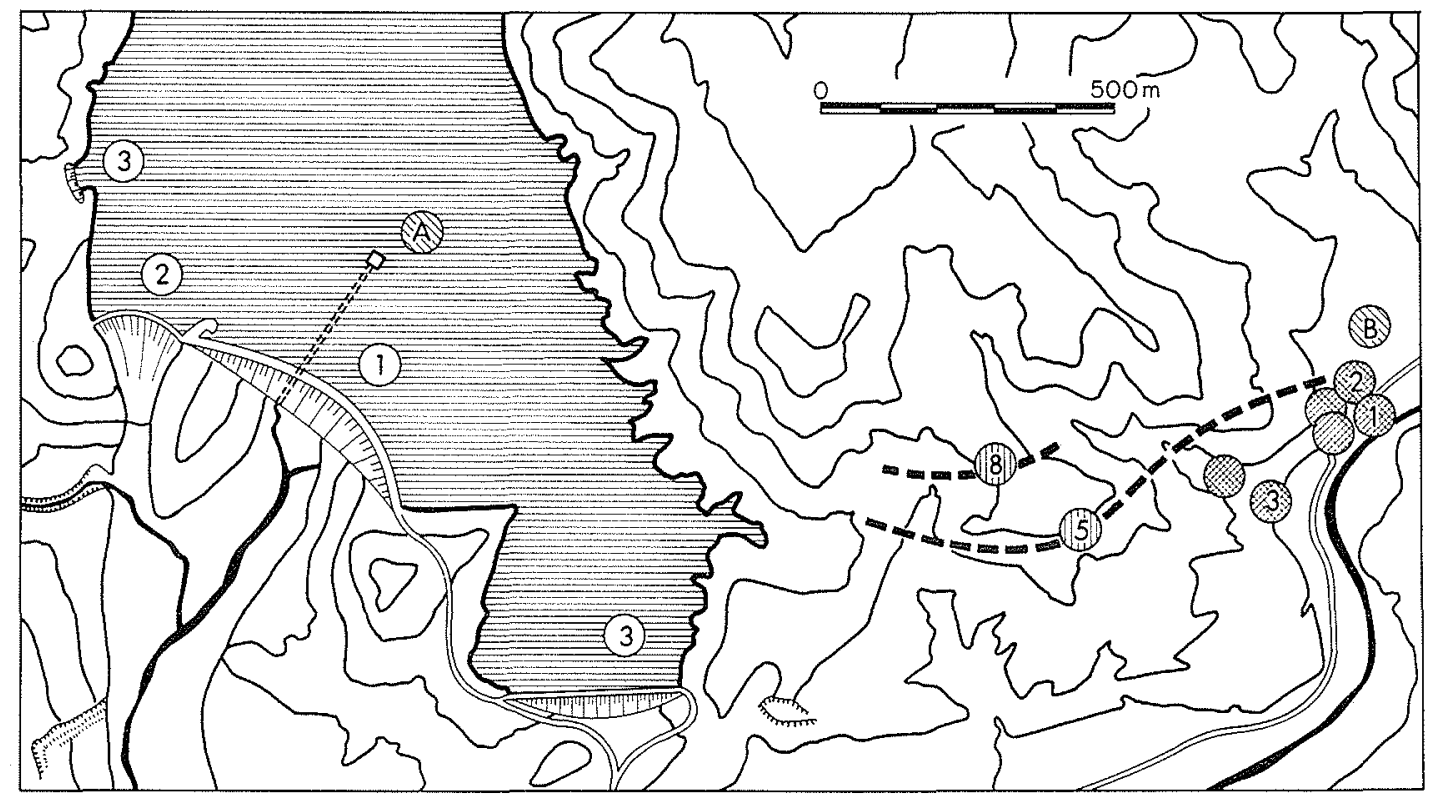

2/ Site de l'ourrage du Nebaana (Tunisie) .

$1 /$ barrage

2/ déversoir - exurgences

$3 /$ digues

4/ tour de prise d'eau

$5 /$ et 6/ failles reconnues ou présumées.

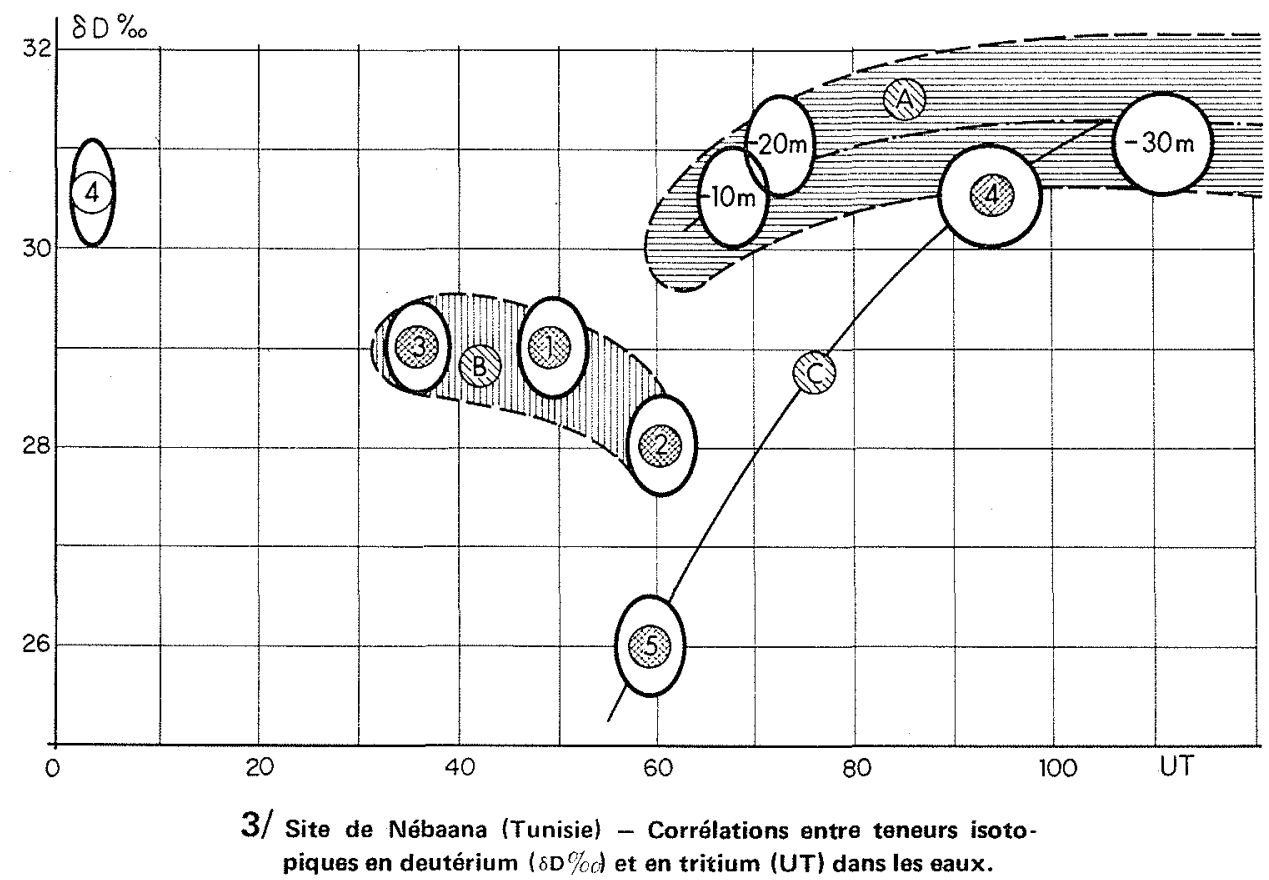

1/ Eaux de la retenue

2/ Groupe des exurgences de l'appui Rive Gauche.

3/ Groupe des exurgences de I'appui Rive Droite (de l'amont vers ['aval)

4/ Aîn et Hammam (source romaine située à $1 \mathrm{~km}$ à l'Ouest de la retenue).

Exurgences (cf. figure 1). 


\section{Mise en œuvre de traceurs artificiels dans la retenue}

Avec les traceurs artificiels, la maitrise du marquage autorise des investigations plus fines et quelquefois des mesures quantitatives de vitesses et de débits d'infiltration.

Les différentes méthodes employées visent tout d'abord à établir la matérialité des circulations, c'est-à-dire à recenser les points d'exurgence, en second lieu à localiser les zones d'infiltration, et enfin, dans des circonstances tout à fait exceptionnelles, à déterminer les trajectoires des circulations préférentielles.

$\mathrm{Au}$ cours de ces mêmes interventions ou au cours d'interventions complémentaires, il est possible d'évaluer les vitesses d'écoulement et de mesurer les débits de fuite effectifs en les distinguant d'apports ne provenant pas du réservoir.

Pour ce qui concerne la technologie des traceurs employés, on se reportera aux références [2] et [6].

\subsection{RECENSEMENT DES EXURGENCES ET EVALUA- TION DE DONNEES HYDRAULIQUES OU HYDRO- DYNAMIQUES PAR MARQUAGE TOTAL OU PAR- TIEL DE LA RETENUE}

\subsubsection{Technologie et méthodologie du procédé}

L'opération de marquage s'effectue en sillonnant méthodiquement la retenue avec une embarcation pourvue d'un dispositif d'injection, de profondeur réglable en fonction du parti adopté, et en tenant compte de la présence éventuelle d'une thermocline. Des considérations pratiques et économiques conduisent, la plupart du temps, à procéder au marquage du culot de retenue, le traceur étant confiné dans l'hypolimnion en présence de thermocline (fig. 4).

Les informations que fournit cette méthode ne devraient pas se distinguer de celles apportées par les mesures de traceurs naturels. En fait, la maîtrise des concentrations en traceurs
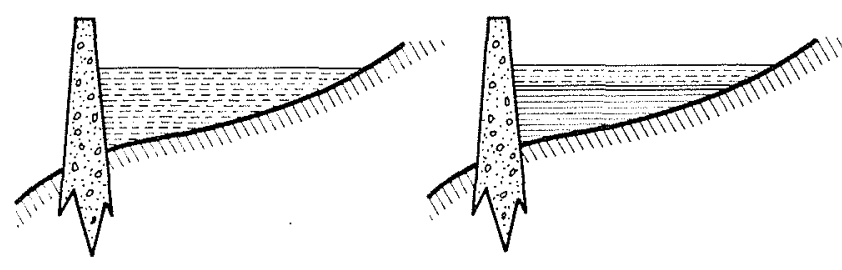

a.

b.

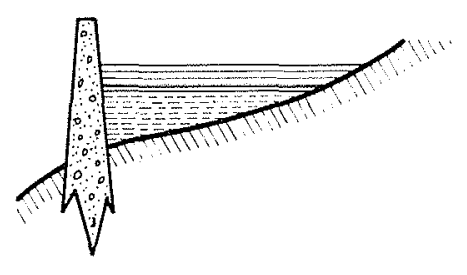

c-

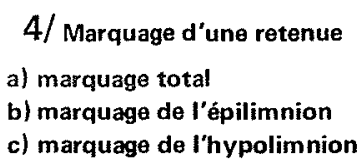

autorise une meilleure résolution. En outre, l'opération de marquage ayant un caractère instantané vis-à-vis des phénomènes à étudier, et l'homogénéité du traceur se révélant généralement satisfaisante, la fonction d'entrée du traceur est bien définie au point qu'il est quelquefois possible de l'assimiler à une fonction "échelon". Dans ces conditions, les courbes de restitution du traceur fournissent, par simple dérivation ou, à défaut après déconvolution par la fonction d'entrée quand celle-ci a une forme arbitraire, la distribution des temps de séjour du ou des système (s).

Enfin, la dilution éventuelle du traceur à l'exutoire permet d'apprécier l'importance d'apports ne participant pas aux circulations concernant l'ouvrage.

4.1.2. Exemples d'observations qualitatives : mise en évidence de circulations ne provenant pas de la retenue et localisation de cotes d'infiltration

Le marquage total de la retenue de St-Mathieu de Tréviers a permis de distinguer, parmi les résurgences, les eaux d'une source pérenne préexistante sans relation avec la retenue [7].

Le marquage du culot de la retenue d'Alfeld (Cf. 4.1.4) a établi que l'alimentation d'une des émergences, exempte de traceur, s'effectuait au niveau de la tranche supérieure de la retenue.

4.1.3. Exemple d'inventaires de fuites et de détermination de caractéristiques de transfert

Le marquage partiel sur une portion limitée par 2 batardeaux (cf. 4.2.1), puis total du bief supérieur du plan incliné d'Arzviller, en contrebas duquel un certain nombre de fuites s'étaient manifestées lors de sa mise en eau, a permis de déterminer :

- les zones où le système de drainage était défectueux,

- le temps de transit de l'eau entre le canal et les exutoires des drains,

- les résurgences qui étaient en liaison souterraine avec le canal,

- le temps de transit de l'eau entre le canal et ces résurgences.

La connaissance des temps de transit s'est révélée importante car elle a permis de caractériser la nature des liaisons, rapide par voies directes ou lente par voies diffuses, les conséquences de ces deux types de fuite n'étant pas identiques (cf. figure 5) [8].

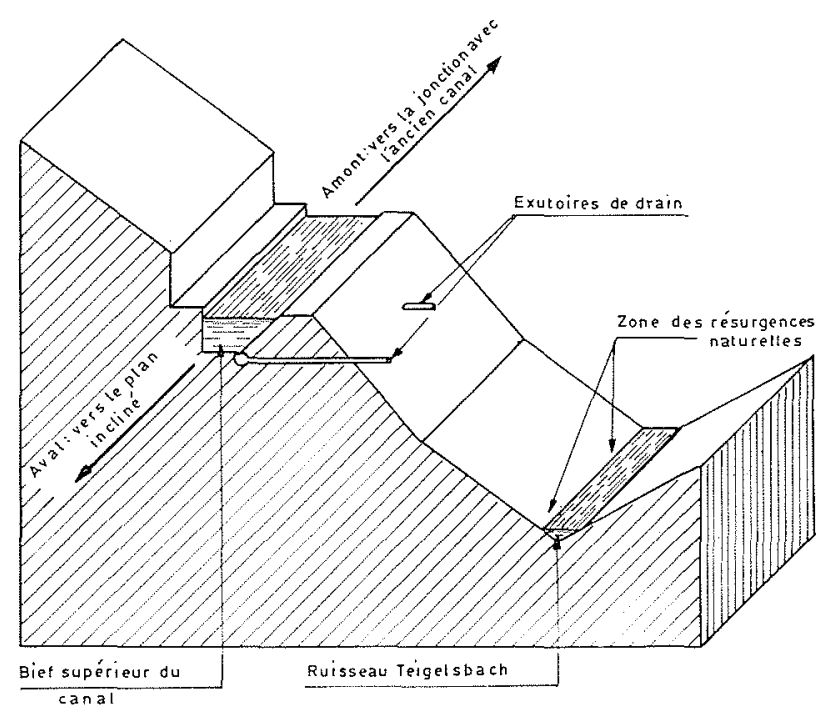

5/ Schéma sommaire situant le bief supérieur du canal et la zone des résurgences naturelles 


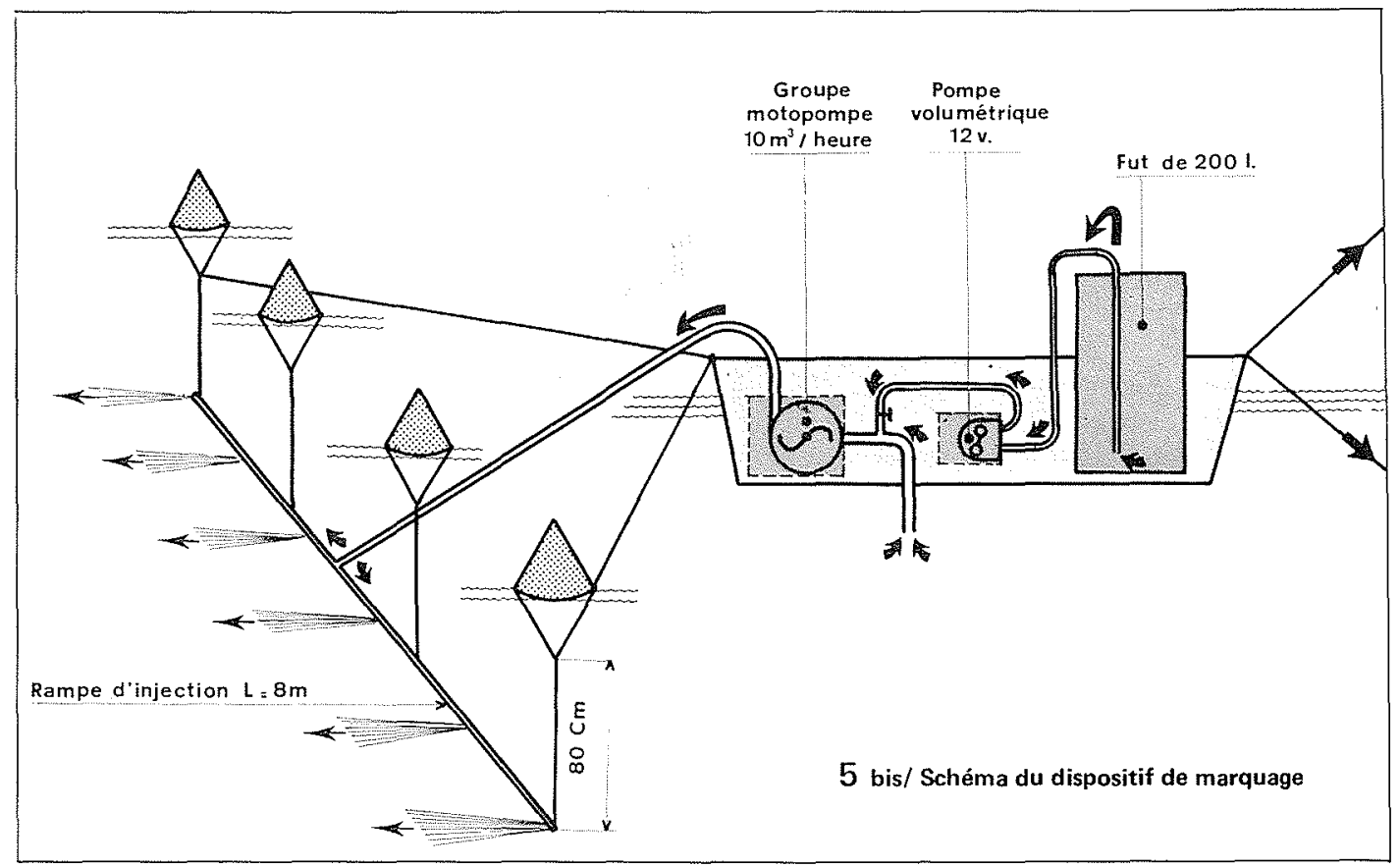

4.1.4. Exemple d'évaluation des débits réels de fuite et des modalités hydrodynamiques de transit à travers l'ouvrage

Le marquage des $250.000 \mathrm{~m}^{3}$ de l'hypolimnion de la retenue d'Alfeld (d'une capacité totale d'environ 1 million de mètres cubes), effectué au moyen d'iodure de sodium, a conduit à une série de résultats extrêmement importants, outre la localisation de cote d'infiltration signalée plus haut (cf. 4.1.2.) :

a) les mesures de concentration en traceur dans les autres émergences ont permis, après établissement du régime permanent en traceur, de préciser les valeurs des débits réels de fuite en les distinguant des apports des eaux météoriques drainées par le massif d'enrochements (Fig. 6).

b) le traitement des données constituées par les courbes de restitution du traceur (fonctions de sortie) et l'évolution des concentrations en traceur dans la retenue (fonction d'entrée) (*) a fourni, par déconvolution de ces couples de fonctions, les réponses impulsionnelles de l'eau, c'està-dire les distributions des temps de séjour (D.T.S.) de l'eau dans chacune des circulations (Fig. 7), D.T.S. à partir desquelles il est possible de calculer les vitesses moyennes et, connaissant le gradient hydraulique, d'approcher une valeur globale des coefficients de perméabilité de Darcy.

\subsection{LOCALISATION DE ZONES D'INFILTRATION PAR CONFINEMENT DE TRACEURS}

Les techniques employées ressortissent de deux procédés : le procédé de confinement du traceur et le procédé que l'on pourrait qualifier de "migration libre".

Dans le premier procédé, on recourt à des confinements d'autant plus discrets et localisés que les informations sur les zones de fuite sont plus précises.

(*) Ires concentrations en traceur dans le culot de retenue ont évolué de façon notable en raison de crues importantes des apports au cours de la période d'observations.

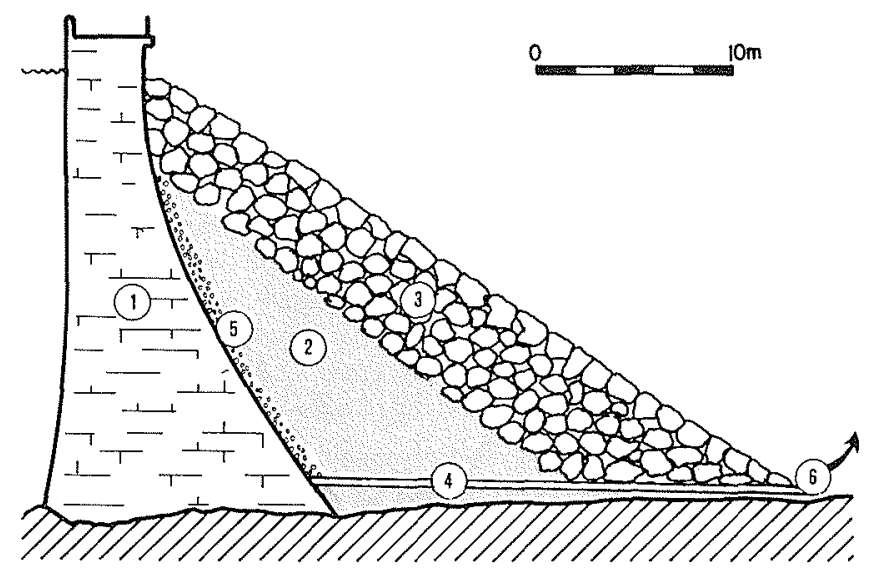

6/

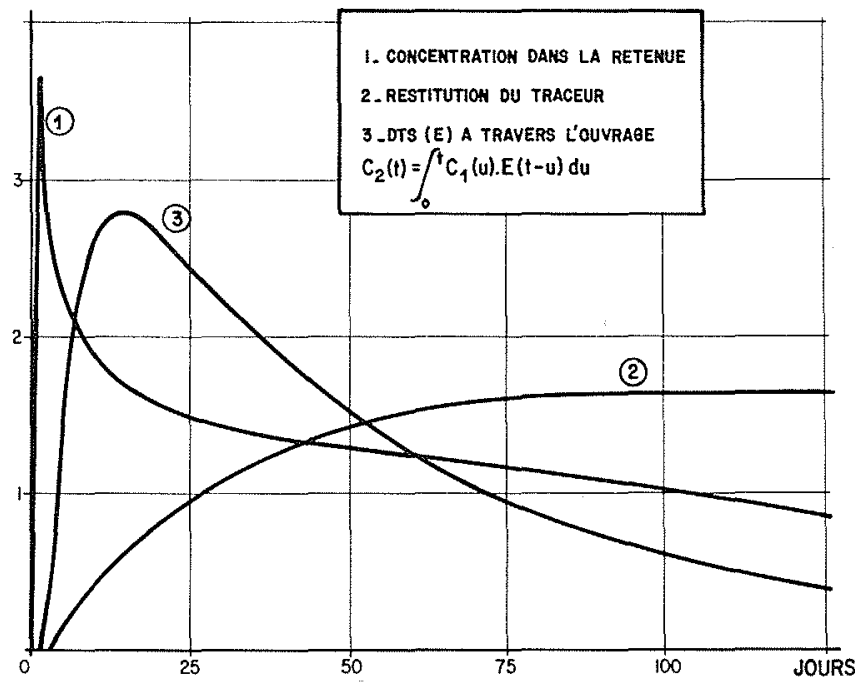

$7 /$ 


\subsubsection{Confinements gravitaires}

La localisation la plus simple et la plus sommaire a été évoquée en (4.1) elle consiste à marquer l'une des couches stratifiées. En l'absence de thermocline, ou pour des marquages de plus faible ampleur, le traceur peut être confiné, par incorporation dans une saumure $\left({ }^{*}\right)$, dans une fosse, une dépression ou un radier.

Dans ce dernier cas, le confinement transversal peut être réalisé grâce à des batardeaux souples immergés, dont la mise au point a été effectuée au SARR [9].

Ce procédé a été notamment employé sur le canal de la Marne au Rhin, à Arzviller (cf. 4.1 .3 et [8]. )

L'emploi de saumure permet aussi d'épandre des coulis (ou rideaux) d'injection pour localiser des fuites sur des plans de parements ou d'appuis d'ouvrages, comme réalisé selon différentes variantes et par utilisation simultanée de différents traceurs radioactifs, chimiques et fluorescents, sur le site de Lastioulles (Fig. 8 et 9) [10].

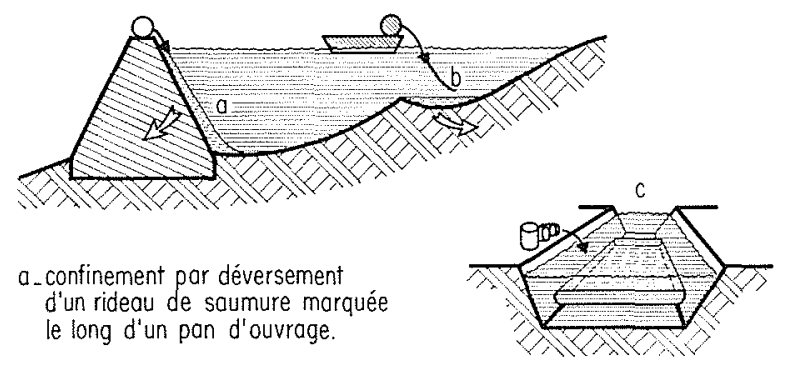

b. confinement d'une saumure marquée c-confinement par batardeaux souples dans une cuvette naturelle. immergés d'une saumure marquée sur un radier de canol.

\section{8/Procédés de confinement par saumures}

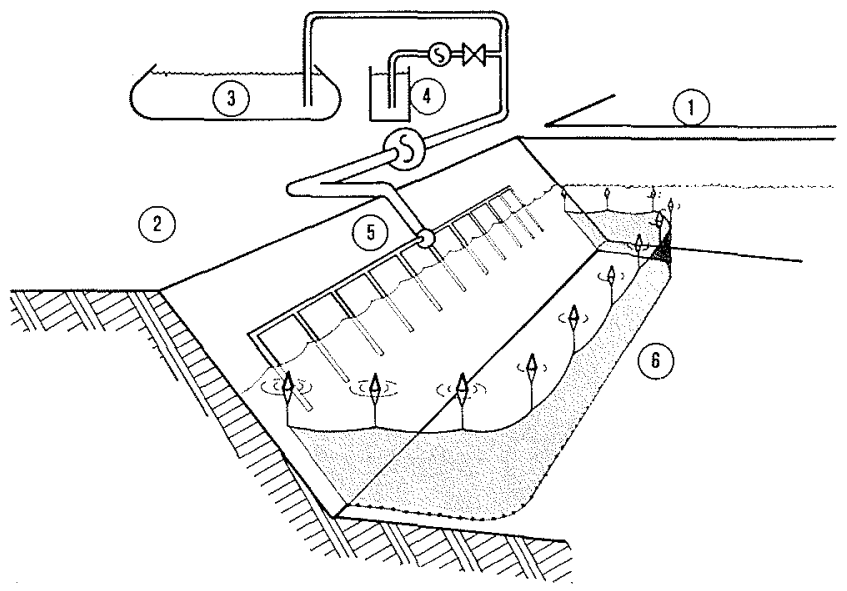

9/Dispositif de confinement mis en oeuvre pour déceler des infiltrations le long de l'appui d'un ouvrage (Lastioulles).

1/ Couronnement de l'ourrage

2/ Appui RG

3/ Réservoir de saumure $\left(10 \mathrm{~m}^{3}\right)$

4/ Solution mère de traceur

5/ Herse de répartition

6/ Voile d'étanchéité faisant obstacle à la diffusion de la saumure marquée vers l'ouvrage et le culot de retenue.

(*) Nos mises au point, comme nos essais réels, ont confirmé que des saumures à $1 \mathrm{~g} / \mathrm{l}$ permettaient d'obtenir un confinement satisfaisant.

\subsubsection{Confinements au moyen de dispositifs spéciaux}

Enfin en recourant aux techniques dites de "l'épervier" ou du "tube filtrant", expérimentées sur le site de Kruth, il devient possible d'injecter le traceur dans des zones restreintes ou en des points précis et de procéder ainsi à des mesures localisées de débits d'infiltration (par la méthode de dilution ponctuelie) et à des mesures globales de transit (fig. 10 et 11) [11] et [12].

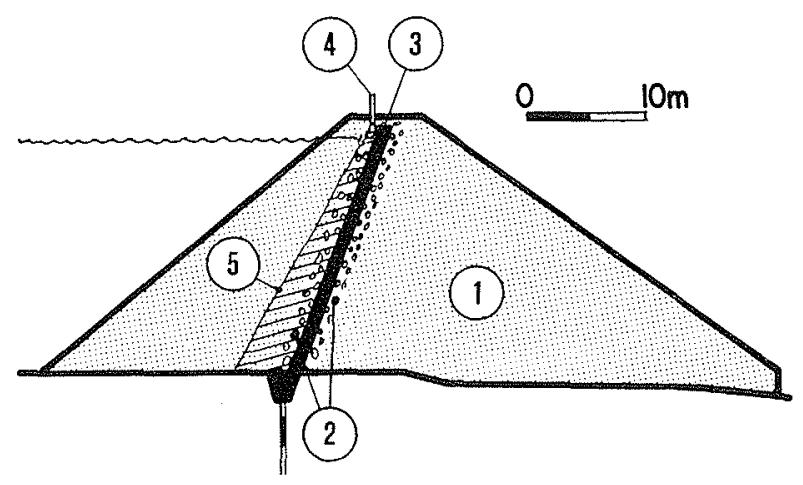

10/ Confinement gravitaire le long du masque d'étanchéité d'un ouvrage en enrochements (Lastioulles)

1/ Enrochements $800 \mathrm{~mm}$

2/ Recharge en matériaux concassés $30-100 \mathrm{~mm}$

3/ Masque d'étanchéité en béton bitumeux

4/ Herse d'injection de saumures marquées

5/ Rideau d'injection.

\subsection{LOCALISATION DE ZONES D'INFILTRATION PAR DES PROCEDES DE LIBRE MIGRATION DE TRA CEUR}

\subsubsection{Méthode du nuage marqué}

Ne citons que pour mémoire le procédé DWICA qui consiste à mesurer, à partir d'une couronne de détecteurs, la direction et la vitesse d'une bouffée de traceur injectée en son centre et ne fournit que des indications très locales sur le mouvement des masses d'eau, lequel ne peut être influencé qu'à proximité immédiate des zones d'infiltration par les courants de fuite.

La méthode dite du "nuage marqué", analogue dans son principe, consiste à effectuer une injection discrète de traceur à proximité d'une zone suspecte. On définit l'évolution de ce nuage dans le temps sous l'influence des courants sublacustres et, en particulier, l'évolution de son interface avec le fond ou les parois de la retenue (Fig. 12). Les singularités de cette évolution pourront être l'indice de zones de fuite dont l'existence et la localisation doivent être confirmées par le contrôle de l'apparition du traceur à l'exutoire des fuites.

Dans ce type d'investigations, on recourt nécessairement à des traceurs radioactifs détectables "in situ", l'intersection du nuage marqué avec le fond étant déterminée à l'aide d'une sonde de détection suspendue à une embarcation dont la trajectoire est repérée par des observateurs servant sur la rive.

Cette méthode a donné des résultats remarquables sur le site du barrage de Kruth-Wildenstein [12] et [13].

\subsubsection{Méthode des natériaux sédimentables en suspension}

Ce procédé, inspiré des processus naturels de colmatage, a été initialement conçu pour l'étanchement de certains 


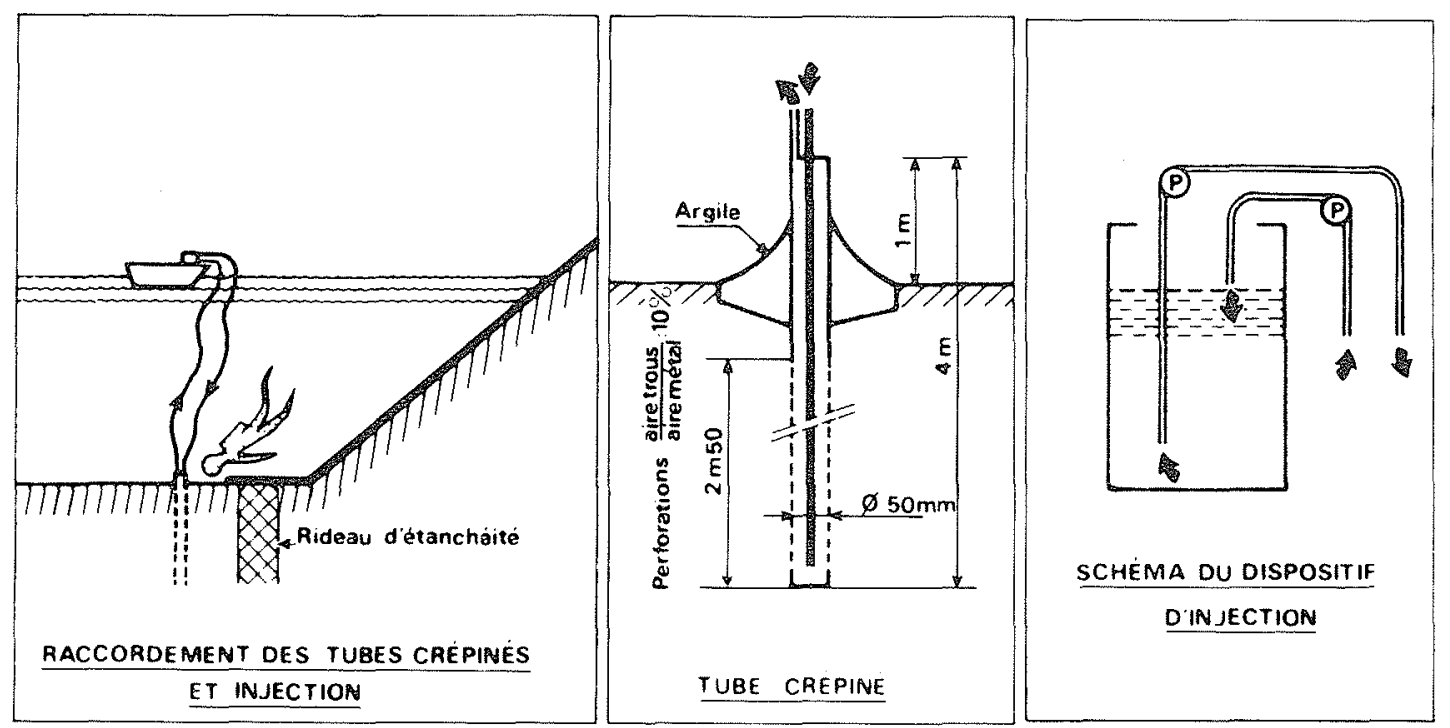

11 / injection de traceur par la méthode du tube filtrant pour la détermination du temps de transit à travers un ouvrage.

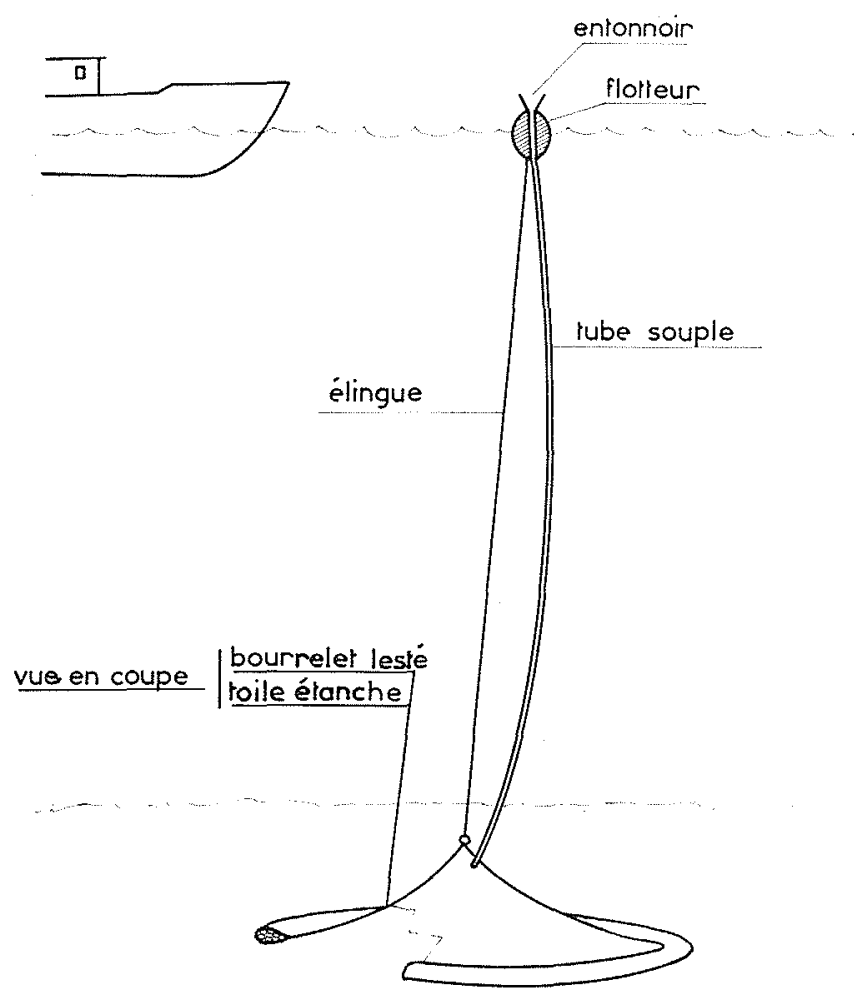

$12 /$ Injection sous enceinte étanche

types de fuites. Il consiste à dispenser des particules de dimensions et de densités leur conférant une vitesse de chute suffisamment négligeable vis-à-vis des courants de fuite. Parmi les particules sélectionnées à l'issue de longues études théoriques et expérimentales [14], les crins en matière plastique colorée se sont révélés d'excellents traceurs pour suivre ces courants et visualiser par observation directe (plongeurs) ou télévision les zones d'entonnement ou d'infiltration. Ce procédé a été appliqué avec succès sur différents ouvrages [15].

\subsubsection{Méthode des émulsions de bitume marquées}

Enfin une technique plus récente dite des émulsions de bitume marquées consiste à dispenser, dans l'ensemble du réservoir ou à proximité de zones présumées suspectes, de fines particules de bitume marquées au moyen de radioisotopes émetteurs gamma.

Au niveau des zones d'infiltration, les particules entraînées par les filets liquides percutent les parois et s'y fixent. La détection de ces zones d'infiltration s'effectue au bout d'un délai suffisamment long pour que la quantité de particules qui s'y sont accumulées soit aisément décelable.

Ce procédé ne s'applique évidemment qu'à des localisations de fuites sur des surfaces régulières (parois, chapes ou revêtements). Il a été utilisé avec succès pour localiser des zones d'infiltration préférentielles sur le radier du canal de la Marne au Rhin à Arzviller (Fig. 13, 14 et 15) avec un dispositif de confinement décrit en 4.2.1. et Fig. 8 [16].

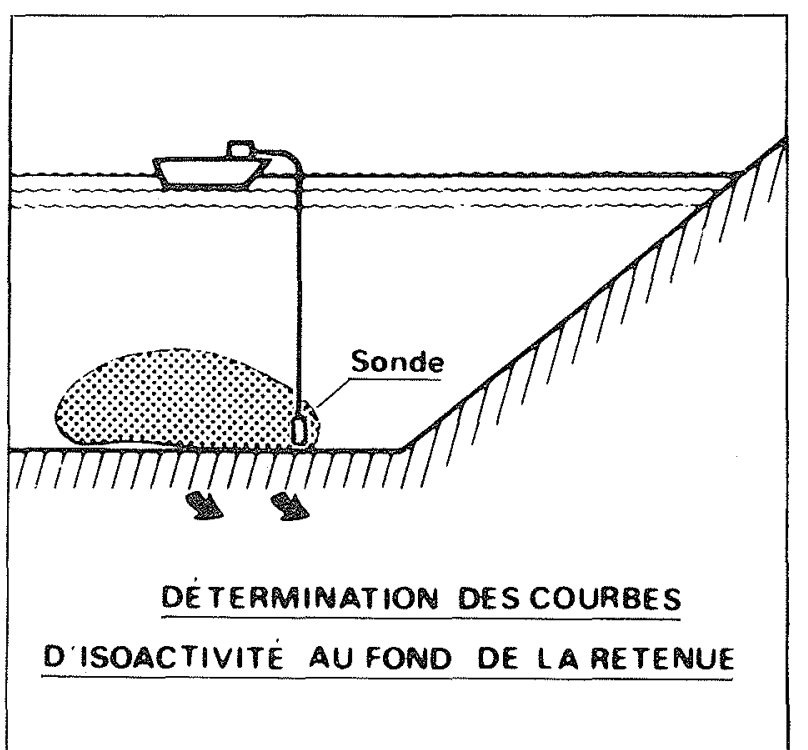

13/ Recherche de fuites dans une retenue par la méthode du nuage 


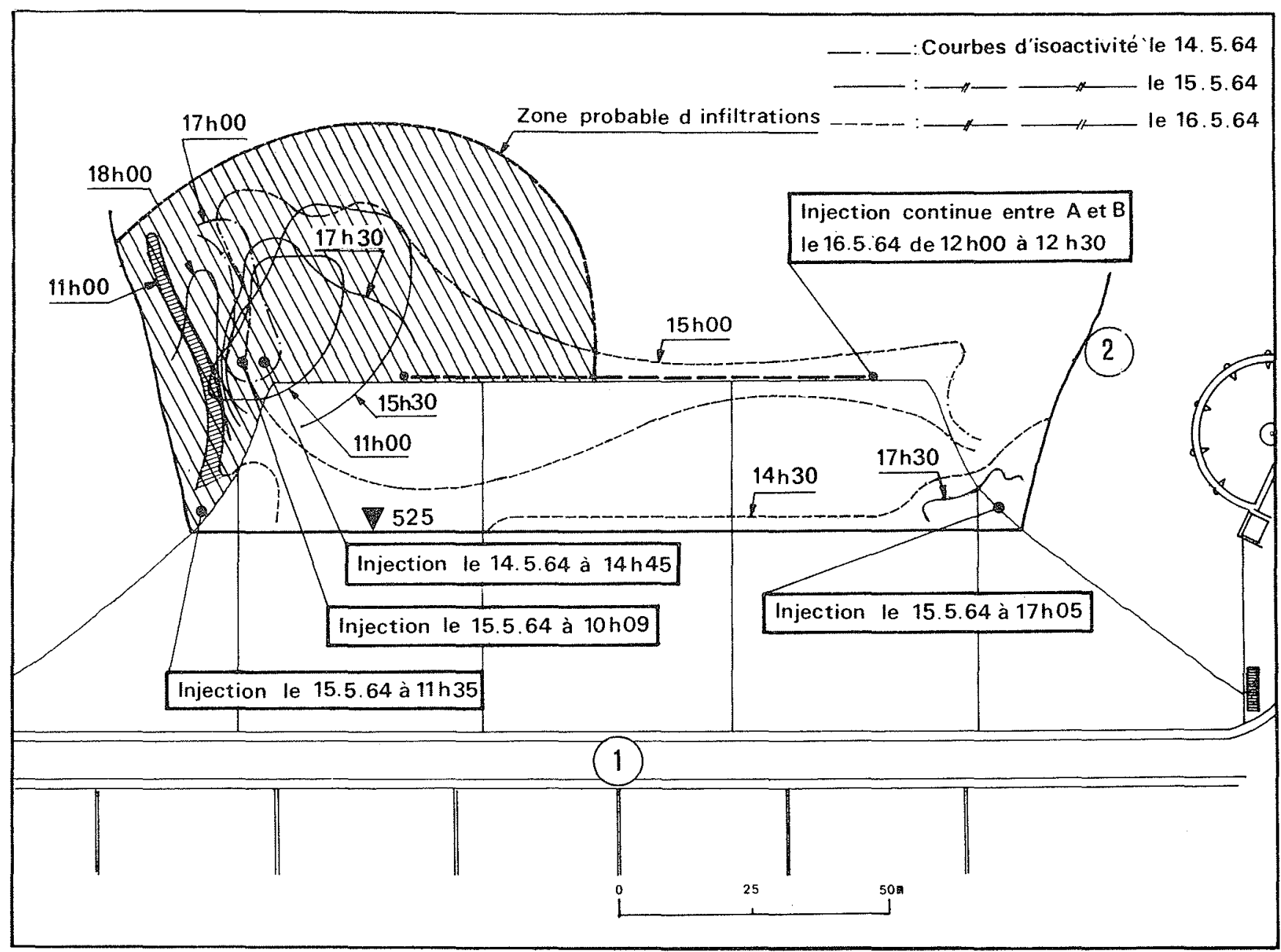

14/ Evolution des courbes d'isoactivité au fond de la retenue (première série d'essais Mai 1964)

Recherche et localisation de fuites par la méthode du nuage marqué (barrage de Kruth)

1 Couronnement de l'ouvrage

2 Limite du plan d'eau

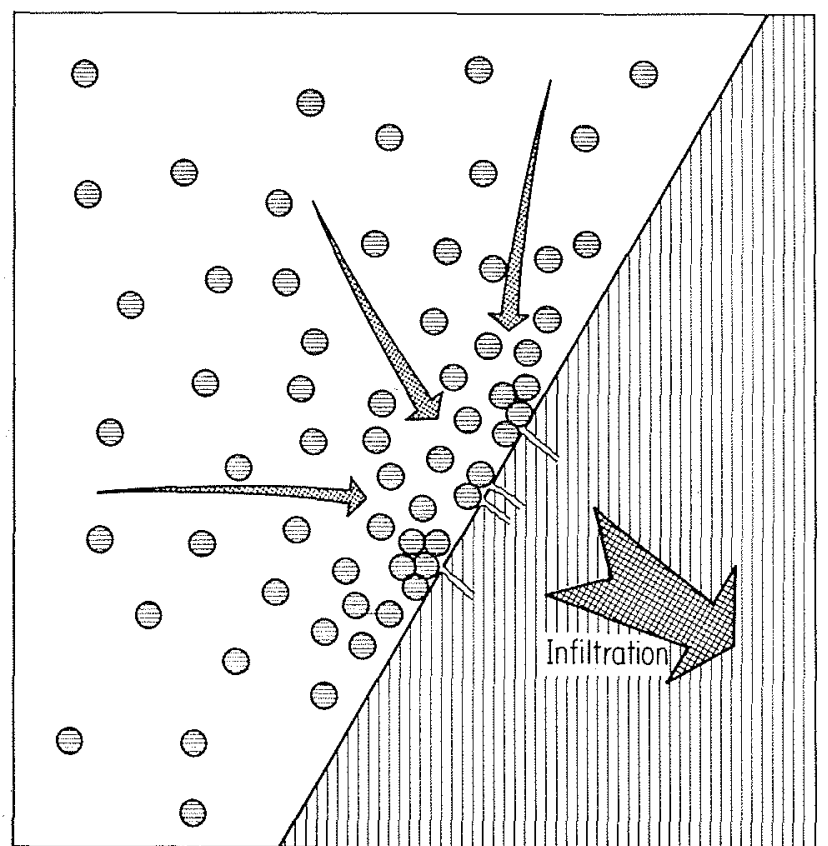

$15 /$ Localisation de zones à perméabilité préférentielle au moyen d'émulsions de bitume marqué

\section{Mise en œuvre de traceurs artificiels dans l'ouvrage et ses appuis}

\subsection{DIAGRAPHIES}

\subsubsection{Profils de vitesses horizontales}

De telles mesures ne peuvent être entreprises que dans des forages non consolidés ou à tubage crépiné. Elles consistent à observer la vitesse de disparition d'un traceur radioactif dans une courte section de forage isolée par des obturateurs [17]. Les mesures effectuées dans l'ouvrage de Kruth et ses appuis (Col du Hof) [13] ont permis de préciser cote et vitesse de circulations préférentielles.

\subsubsection{Autres mesures}

Dans ces mêmes forages il est possible de procéder à des profils d'injectivité ou, à l'aide d'un appareillage approprié, des mesures de directions d'écoulement [2]. Ce type de mesures reste néanmoins d'utilisation peu courante. 

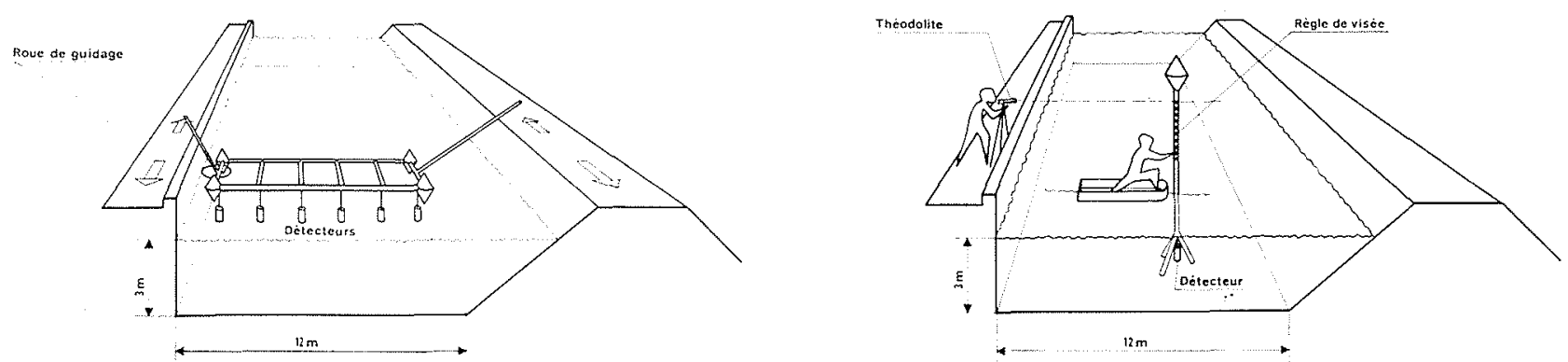
$16 /$ a) Schéma du dispositif (herse) d'exploration systématique
b) Exploration détaillée d'une zone au moyen d'une sonde
montée sur trépied.

\subsection{DETERMINATION DE TRAJECTOIRES DE CIRCU- LATIONS PREFERENTIELLES}

La détermination des trajectoires ne peut être entreprise qu'à condition de disposer de points d'accès à l'horizon aquifère en nombre suffisant pour procéder à une reconstitution itérative des circuits de transits. Elle est envisageable lorsque s'offre la possibilité de procéder à des forages peu coûteux (sur des ouvrages ou appuis de hauteur modérée) ou de tirer parti d'un réseau de forages exécutés en vue d'injections de consolidation ou d'étanchement.

Cette recherche itérative s'effectue généralement à partir du ou des exutoires et par injections successives dans des points d'accès de plus en plus éloignés des exurgences. Ce procédé ancien, aujourd'hui perfectible par multitraçages, devrait être utilement complété par une méthode mixte reposant sur la détection par conductivité de courants de saumure injectée dans la retenue [18].

\section{Conclusions}

La recherche de l'origine des infiltrations dans une retenue revet un caractère d'autant plus crucial que l'importance des fuites est limitée à la fois par des impératifs de sécurité et des considérations économiques qui rendent préoccupants, non seulement le volume global des pertes, mais aussi les vitesses de circulation, lesquelles ne peuvent dépasser certaines valeurs pouvant entraîner, par exemple, la formation de renards capables de mettre en cause la sécurité des ouvrages et de leurs fondations.

Parmi les procédés permettant de préciser l'origine des infiltrations et de surveiller leur évolution, les techniques de traceurs offrent, dans leur extrème spécificité, des moyens d'investigation souvent sans équivalents.

Il apparaît toutefois nécessaire, pour la mise en oeuvre des techniques précédemment décrites, d'attirer l'attention sur les points suivants :

a) Les techniques de traçage relèvent d'une systématique et non de l'improvisation. Un excès de colorant mis en place lors des recherches préliminaires peut, par exemple, compromettre les recherches ultérieures. Une utilisation intempestive de traceurs radioactifs de longue période peut également condamner un site en s'opposant à la réitération d'un essai et en occultant toute investigation sur les radioisotopes du milieu [20]. b) Les recherches d'infiltration, après mise en service des ouvrages, seraient largement facilitées si les projeteurs, lors de la conception, songeaient aux éventuelles difficultés d'exploitation et prévoyaient la mise en place, au cours de l'édification de l'ouvrage, de dispositifs appropriés au cas considéré (tubes filtrants, tubes crépinés, compartimentage des réseaux de drainage, dispositifs de mesure, etc...). On devrait aussi toujours songer à constituer une banque d'échantillons de l'eau du site pour des études ultérieures d'environnement dès avant les opérations de mise en eau [20].

c) Les exploitants de barrages ne doivent pas oublier qu'il existe aujourd'hui de multiples techniques et notamment des méthodes de traçage susceptibles de leur apporter un précieux concours dans les cas les plus difficiles. Leur mise en cuvre peut contribuer à maintenir un niveau satisfaisant dans la sécurité d'exploitation durant la vie des ouvrages hydrauliques.

\section{Références}

[1] CARLIER (M.) - Inspection et surveillance des barrages - Bulletin Technique du Génie nural n. SI 68 - Ministère de l'Agriculture - CERAFER - Antony - (1969) 19 pages.

[2] MOLINARI (J.) - Les traceurs isotopiques en hydrologie - Annales des Mines, Oct. 1969, pp. 13-38.

[3] Cornuet (R.), Courtois (G.), Gras (R.), Guizerix (J.), MARGRita (R.). MERlivat (L.), MOLINARI (J.), ROTH Application des isotopes stables et radioactifs dans le domaine de l'hydrologie et de la sédimentologie - Peaceful Uses of A tomic Energy , United Nations - New-York/AEIA Wien 1972, 14, pp. 425-440.

[4] MOlinari (J.), Corompt (P.), CAlmels (P.), Protche (G.) Etude des corrélations entre la retenue d'Alfeld et les émergences situées au pied de l'ouvrage - Commissariat à l'Energie Atomique - Centre d'Etudes Nucléaires de Grenoble - Rapport SAR-G/7118, Sept. 1971, 96 pages, $40 \mathrm{pl}$.

[5] MOLINARI (J.) - Etude du site du barrage de l'Oued Nebaana. Commissariat à l'Energie Atomique - Centre d'Etudes Nucléaires de Grenoble - Rapport SAR-G/70-21, juin 1970, 9 pages, $2 \mathrm{Tabl}$ $7 \mathrm{pl}$.

[6] MOLINARI (J.) - Les traceurs salins et fluorescents en hydrologie - Aspects techniques et économiques de leur utilisation. Commissariat à l'Energie Atomique - Centre d'Etudes Nucléaires de Grenoble - Rapport SAR-G/69-15, juin 1969,36 pages.

[7] MOLINARI (J.), CAlmElS (P.) - Recherche et localisation de fuites sur la retenue de fontaine de Jeantou. Procès verbaux des essais - Commissariat à l'Energie Atomique - Centre d'Etudes Nucléaires de Grenoble - Rapports SAR-G/74-30, Nov. 1974 4 pages, 12 planches et SAR-G/75-05, mai 1975, 17 pages, 13 planches. 
[8] Molinari (J.), Calmels (P.), Millet (M.) -- Recherche de fuites sur le bief supérieur du plan incliné de Saint-Louis Arzviller. Commissariat à l'Energie Atomique - Centre d'Etudes Nucléaires de Grenoble - Rapport SAR-G/69-02, Fév. 1969, 28 pages, 4 tabl, $25 \mathrm{pl}$

[9] GAILLARD (B.), CALMELS (P.) - Etude et mise en ouvre d'un écran de confinement d'une solution dense marquée pour l'application d'une méthode de recherche de fuites.- Commissariat à l'Energie Atomique -. Centre d'Etudes Nucléaires de Grenoble Rapport SAR-G/69-26, Octobre 1969, 4 pages, 2 pl.

[10] MOLINARI (J.) et Collaborateurs - Compte rendu des essais de localisation de fuites sur l'ouvrage de Lastioulles-Nord -Commissariat à l'Energie Atomique - Centre d'Etudes Nucléaires de Grenoble - Rapport SAR-G/70-31, Déc. 1970,34 pages, 4 Tabl. 26 pl., 9 clichés.

[11] MOLiNARI (J.) - Mise en ouvre subaquatique de la méthode du tube filtrant pour la recherche de fuites dans les réservoirs et les canaux-Commissariat à l'Energie Atomique - Centre d'Etudes Nucléaires de Grenoble - Rapport SAR-G/67-32, Août 1967, 24 pages, $17 \mathrm{pl}$.

12] Guizerix (J.), MOlinari (J.), GAIllard (B.), CORdA (R.) Localisation de fuites sur un grand réservoir à l'aide de traceurs radioactifs - Isotopes in Hydrology - Agence Internationale de l'Energie Atomique, Wien 1967, pp. 587-599.

[13] CORDA (R.), RingENBACH (P.), GUIZERIX (J.), MOLINARr (J.), BOLLO (MF.) - Localisation et contrôle des circulations d'eau dans les fondations, la digue et les appuis du barrage de Kruth Wildenstein - Commission Internationale des Grands Barrages $10^{e}$ Congrès des Grands Barrages. Montréal 1970, Q 37 R45, pp. 841-871.
[14] PATiN (P.) - Un nouveau procédé de repérage et de colmatage des fuites dans les réserves d'eau - Supplément aux Annales de l'Institut Technique du Bätiment et des Travaux Publics $1 \mathrm{n}^{\circ} 253$ Questions générales 101 (1969), 20 pages.

[15] BORDES, PATIN (P.), BARBEDETTE (R.) - Commission Internationale des Grands Barrages - $10^{e}$ Congrès des Grands Barrages, Montréal 1970, Compléments au R 23 Q 37, p. 402

[16] MOLINARI (J), GUizERIX (J), CHAMBARD (R.) - Nouvelle méthode de localisation de fuites sur des réservoirs ou canaux au moyen d'émulsions de bitume marqué - Isotope Hydrology 1970 - Agence Internationale de l'Energie Atomique, Wien 1970,pp. 743-760.

[17] GUIZERIX (J.), GRANDClement (G.), GAillard (B.), RUBY (P.) -- Appareil pour la mesure des vitesses relatives des eaux souterraines par la méthode de dilution ponctuelle-- $R a$ diosotopes in Hydrology - Agence Internationale de l'Energie Atomique, Wien 1963 pp. 25-35.

[18] LAURENT (J.) - Recherches expérimentales des causes, des origines et des trajets de fuites souterraines. Application à un canal de navigation-Revue Générale de l'Hydraulique, 1949 $\mathrm{n}^{\circ} 50$ et 51

[19] Groupe de Travail du Comité Français des Grands Barrages BARBEDETTE (R.), BOLlLO, CORDA (R.), DENISE (J.P.), GUIZERIX (J), LURIN (P.), MOLINARI (J.) - La recherche de l'origine des infiltrations des barrages et de leurs fondations. Evaluation des fuites d'un réservoir perméable - Commission Internationale des Grands Barrages. $12^{e}$ Congrès des Grands Bar rages Mexico 1976 Vol. 2 Q 45 R 10 pp. 675-703.

[20] MOLINARI (J.) -... Interventions sur les Questions 45 et 46 Commission Internationale des Grands Barrages. $12^{e}$ Congrès des Grands Barrages. Mexico 1976. Vol. 5 (sous presse)

\section{Discussion}

Président : M. Roger ROCHE

M. Le Président remercie M. MOLiNAR I de son exposé qui intéressera de nombreux spécialistes des aménagements hydrauliques. Il ouvre ensuite le débat.

M. le Professeur Paul-Ch. Leveque signale que les banques d'échantillons souhaitées par le Conférencier fonctionnent depuis quelques années, notamment en Suisse où la construction de quelques grands barrages est encore envisagée (Glacier du Rhône, Grimsel-Furka, Jura Suisse).

A cette occasion, il indique que les prises d'échantillons doivent être effectuées en flacon de verre ; certains flacons de polyéthylène donnent lieu à des phénomènes de diffusion qui entrainent une diminution assez sensible des teneurs en tritium et surtout de deuterium et d'oxygène 18 .

M. REMENIERAS intervient, en ces termes:

"M. MOLINARY a parlé de stratification thermique dans les retenues en général bien nette dans les lacs naturels, cette stratification est souvent très perturbée dans les réservoirs créés dans une vallée par l'implantation d'un barrage ; et ce, d'autant plus que le débit arrivant par l'amont est important par rapport au volume du dit réservoir (influence du taux de renouvellement des eaux). Les expériences utilisant des traceurs, évoquées par le Conférencier, ont-elles éclairci le mécanisme et la nature de ces perturbations?"

En effet, répond M. MOLINARI, l'expérience confirme l'existence de ces perturbations, qui ne se manifestent que de manière transitoire, sans affecter (sauf pour les retenues à coefficient de remplissage élevé) la stra. tification. Une certaine stratification se maintient généralement et il faut toujours en tenir compte dans les directives d'échantillonnage ou les interventions. Notons d'ailleurs qu'il serait plus correct, surtout dans le cas de réservoirs de barrages, de parler de stratification de densité que de stratification thermique ; en effet, les teneurs en matières en suspension et en sels -variables avec la saison-interviennent concurremment avec les fluctuations de la température de l'eau.
Dans une intervention qu'il a résumée comme suit, $M$. BARBEDETTE (Sté BACHY) donne ensuite d'intéressants renseignements sur le procédé P.T.M. : évoqué dans l'exposé de M. Molina RI :

Ce procédé $\left(^{1}\right)$ a effectivement été imaginé originellement, dans un but essentiel de colmatage des fuites dans les réservoirs d'eau naturelles ou artificielles ; je rappelle qu'il consiste à disperser dans l'eau des particules de dimensions et densité telles que leur vitesse de chute soit suffisamment faible pour qu'elles soient entraĩnées par les courants de fuite.

"Des études théoriques et expérimentales, longues et délicates, ont permis de sélectionner une gamme de matériaux à choisir en fonction des dimensions des vides à colmater :

- Eléments supérieurs à $10 \mathrm{~mm}$ : béton cellulaire

- Eléments de 10 à $10^{-1} \mathrm{~mm}$ : granulés et crins de plastique

- Produits de finition : cellulose, bentonite, émulsions de bitume ou de latex, fllaments de bitume.

"Les crins de plastique colorés se sont révélés être d'excellents traceurs, l'observation se faisant directement (plongeurs) ou par télévision ils ont notamment été utilisés avec succès lors d'une campagne de repérage des fuites au lac d'IlıKı (Grèce, 1965), lac situé en site karstique et constituant l'une des sources d'alimentation en eau de la ville d'ATHENES

"Depuis, cette méthode des crins colorés a été appliquée plusieurs fois en Tchécoslovaquie ; notamment au barrage en enrochements de NYRSKO où l'on a pu ainsi déceler des joints ouverts et des fissures du masque en béton en partie inférieure de ce dernier, et autour de la tourdéversoir qui traverse ce masque ; les nuages de crins étaient créés selon

(1) Procédé breveté PATIN, TER-MINASSIAN ; cf. ANNALES I.T.B.T.P.I./ 1969, supplément 253 : "Un nouveau procédé de repérage et de colmatage des fuites dans les réservoirs d'eau". 
un quadrillage ; comme à lLıKI, on utilisa, en outre, des "balais de sorcière" (chevelures de crins longs s'orientant dans les courants) ; mais à NYRSKO, ces balais furent implantés à la base du masque et leur observation fut rendue difficile par la turbidité de l'eau dans cette zone. Les crins mesurent habituellement $0,12 \mathrm{~mm}$ de diamètre et 10 à $12 \mathrm{~mm}$ de longueur; des crins fluorescents facilitent l'observation.

"Des communications ont été faites sur ce sujet au dixième Congrès International des Grands Barrages $\left({ }^{2}\right.$ ) (MON T REAL, 1970).

"Le procédé P.T.M., observe M. REMENIERAS, fait penser à un artifice utilisé il y a quelques dizaines d'années par un ingénieur praticien pour localiser les fissures du revêtement d'une galerie sous pression ; ces fissures s'ouvraient et donnaient lieu à des fuites lorsque l'ouvrage était en service ; elles se refermaient et devenaient peu visibles lorsque la galerie était vidée pour visite et réparation. Pour visualiser l'emplacement des fissures dans ces dernières conditions, on dispersait dans l'eau à l'entrée de la galerie en fonctionnement du foin haché fin (les crins de matière plastique n'existaient pas à cette époque). Après un temps suffisant et moyennant un peu de chance, des brins de foin étaient entraînés vers les fissures et plus ou moins piégés par celles-ci rendant ainsi bien visible le tracé des fissures lors de la visite de la galerie".

En l'absence d'autre intervention, M. Le Président lève la séance en remerciant au nom de la Société Française d'Energie Nucléaire et au nom de la Société Hydrotechnique de France, tous ceux qui ont contribué au succès de la Session commune sur les traceurs en hydrologie et en hydraulique.

(2) "Réalisation des barrages et des retenues en pays karstique ; tendances françaises actuelles" (Groupe de travail du Comité français des Grands Barrages $-\mathrm{R} .23, \mathrm{Q} .37$ - cf. page 402)

Complément au rapport précédent (communication après congrès : BORDES, PATIN, BARBEDETTE). 